\title{
Health Status as Result of Analyses of Parameters of Hydrogen Bonds Among Water Molecules in Human Body. Entropy in Living Organisms
}

\author{
Ignat Ignatov \\ DSc, Professor, Scientific Research Center of Medical Biophysics (SRCMB), \\ N. Kopernik Street, 32, Sofia 1111, Bulgaria
}

\begin{abstract}
Mankind has always been excited about longevity. Biophysical studies indicate that the water in the human body "has departed" from the water at the time of origination of life (Ignatov, 2010; Mosin, Ignatov, 2011). The more rapid the life processes are, the faster the states of orderliness are observed, i.e. entropy in the living organism decreases. Yet this leads to difficulties in compensating entropy with the entropy of the environment, which is associated with metabolism and energy. Such organisms, like mammals, can live up to 100 years. In trees the processes are slower, the states of orderliness are harder to obtain, and entropy decreases more slowly than in animals. There are trees that live for more than 1000 years. For example, a turtle's life processes are slower than those of mammals and faster than those of trees. It can live up to 300 years. We can define a specific time for each living creature, and their time to a certain degree differs from the time of the environment. This time correlates with parameters of the vital activity of living organisms.Aging is associated with accumulation of errors in DNA replication. The free radicals have an adverse affect on this process. Aging is a non-linear process. The rate of aging increases with time.This paper shows that mountain and melt water is among the most important factors for health and longevity. Other factors are - hereditary, gender, body weight, food, movement, psychological status, family relationships.
\end{abstract}

Keywords: entropy, longevity, spectral analyses, NES, DNES.

DOI: $10.7176 / \mathrm{JMPB} / 64-03$

Publication date: February $29^{\text {th }} 2020$

\section{Introduction}

Entropy is a measure of randomness or disorder of the physical system. It is expressed in the number of possible arrangements of the components. Prigozhin received the Nobel Prize explaining that at a statistical level the chaotic states of living systems lead to an irreversible behavior. Self-structuring and self-organization are observed. He explains the auto-oscillatory reaction of Belousov-Zhabotinsky. Prigozhin shows how together with the increase of entropy, self-organization originates. According to the author, living organisms decrease their own entropy due to their orderliness (Ignatov, 2011). This orderliness increases with the transition from unicellular to multicellular organisms. Cells divide in a particular sequence. Living organisms live with their our own energy, and also exchange substances and energy with the environment. The environment increases its entropy and thus its disorder. Over time, the living organism has more and more difficulties to adapt. This adaptation depends on the consistency and velocity of life processes.

Natural waters derived from various Bulgarian water springs. The blood serum of cancer patients between 50 and 70 years old were investigated by IR, NES and DNES-methods. The control group is with healthy people between 50 and 70 years old. As estimation factor was measured the values of the average energy of hydrogen bonds $\left(\Delta \mathrm{E}_{\mathrm{H} \ldots \mathrm{O}}\right)$ among $\mathrm{H}_{2} \mathrm{O}$ molecules, as well as local extremums in DNES- and IR-spectra of various samples of water and human blood serum, with basic local extremum detected at $\mathrm{E}=-0.1387 \mathrm{eV}$, wavelength $\lambda=8.95 \mu \mathrm{m}$ and wavenumber $\tilde{v}=1117 \mathrm{~cm}^{-1}$. For a group of people in critical condition of life and patients with malignant tumors the greatest values of local extremums in NES- and IR-spectra are shifted to lower energies relative to the control group. The IR-spectrum of mountain water is most similar to the IR-spectrum of blood serum of healthy group of people with a local maximum at $\lambda=8,95 \mu \mathrm{m}$ (Ignatov, Mosin et al., 2014).

\section{Materials and Methods}

\subsection{Spectral analyses of water with methods NES and DNES}

The energy spectrum of water is characterized by a non-equilibrium process of water droplets evaporation; therefore, the term non-equilibrium spectrum (NES) of water is used. The difference

$\Delta f(E)=f($ samples of water $)-f$ (control sample of water $)-$

is called the "differential non-equilibrium energy spectrum of water" (DNES)

The Figure 1 shows that on the $\mathrm{X}$-axis are depicted three scales. The energies of hydrogen bonds among $\mathrm{H}_{2} \mathrm{O}$ molecules are calculated in electronvolts $(\mathrm{eV})$. On the $\mathrm{Y}$-axis is depicted the function of distribution of $\mathrm{H}_{2} \mathrm{O}$ molecules according to energies $\mathrm{f}(\mathrm{E})$, measured in reciprocal electronvolts unit $\mathrm{eV}^{-1}$. The local extremums of 
water samples are detected at $\mathrm{E}=-0.1112 \mathrm{eV}, \mathrm{E}=-0.1212 \mathrm{eV}$ and $\mathrm{E}=-0.1387 \mathrm{eV}$. The value measured at $\mathrm{E}=-$ $0.1212 \mathrm{eV}$ is characteristic for anti-inflammatory effect (Ignatov, Gluhchev, Karadzhov et al., 2014). The value measured at $\mathrm{E}=-0.1112 \mathrm{eV}$ is characteristic for the presence of $\mathrm{Ca}^{2+}$ ions in water (Antonov, 1995). The value measured at $\mathrm{E}=-0.1387 \mathrm{eV}$ is characteristic for inhibiting the growth of tumor cells (Ignatov, Mosin, 2012). Experiments conducted by Antonov with cancer cells of mice in water demonstrated a reduction of this local extremum to a negative value in DNES spectra.

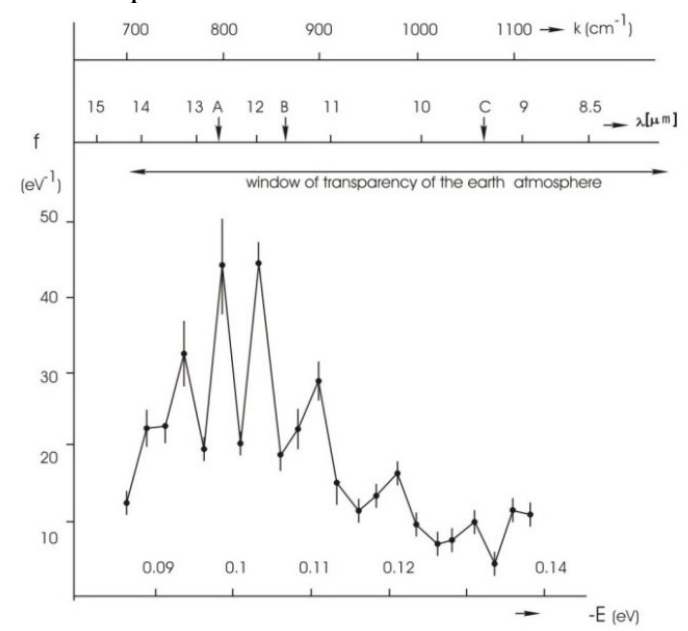

Figure 1. The NES-spectrum of deionized water (chemical purity - 99.99\%; $\mathrm{pH}-6,0-7,5$; electric conductivity $-10 \mu \mathrm{S} / \mathrm{cm})$ : the horizontal axis shows the energy of the H...O hydrogen bonds in the associates $(-\mathrm{E}(\mathrm{eV}))$; the vertical axis - the energy distribution function $-\mathrm{f}\left(\mathrm{eV}^{-1}\right) ; k$ - the vibration frequency of the $\mathrm{H}-\mathrm{O}-\mathrm{H}$ atoms $\left(\mathrm{cm}^{-1}\right)$; $\lambda$ - wavelength $(\mu \mathrm{m})$

\subsection{Groups for research}

$\mathbf{1}^{\text {st }}$ group (group) The control group is with healthy people between 50 and 70 years old $2^{\text {nd }}$ group The research group is with people with ontological diseases.

3d group The groups of brothers and sisters of long-living people.

The research of blood serum is without information of the name of the people. The serums are with codes.

\subsection{Studying the Human Blood Serum}

$1 \%(\mathrm{v} / \mathrm{v})$ solution of human blood serum was studied with the methods of IR-spectroscopy, non-equilibrium (NES) and differential non-equilibrium (DNES) spectral analysis. The specimens were provided by Kalinka Naneva (Municipal Hospital, Bulgaria). Two groups of people between the ages of 50 to 70 were tested. The first group (control group) consisted of people in good clinical health. The second group included people in critical health or suffering from malignant diseases. The third group is of brother and sisters of long-living people.

\subsection{Statistical Processing of Experimental Data}

Statistical processing of experimental data was performed using the statistical package STATISTISA 6.0 using the Student's $t$ - criterion (at $\mathrm{p}<0.05$ ).

\section{Results and Discussions}

\subsection{Entropy in living matter}

The Russian scientist Semihina studied the tangent of dielectrical losses physical indicator for water in different animals (Semihina, 2006). Names of animals in the figure 2 from top to bottom - earth-worms (1), carassius fish (2), mouse (3), frog (4), hamster (5). The largest the extremities in this parameter, especially at $200 \mathrm{KHz}$ or in the kilometer range of the e. $\mathrm{m}$. waves, the highest level of evolutionary development of the animal.

This is also an indicator for the "distancing" of the water in the different animals from the initial water for the origination of life (Ignatov, 2010; Ignatov, Mosin, 2013). This is also essential evidence that water is diverse in the various living creatures. When testing water in animals, there are differences in comparison with water in plants and natural waters. In animals bioelectric processes are more dynamic compared to plants. Mineral water, which interacts with calcium carbonate and sea water, is tested as a model system. Therefore it is difficult to make conclusions about the structure of water from bioelectrical indicators in animals without a parallel spectral analysis. Semihina demonstrates results with a frog. When there is stress, one can observe a peak at $200 \mathrm{KHz}$, in a tranquil state the peak decreases. Additional tests are required in order to prove whether stress influences the structure of water. 


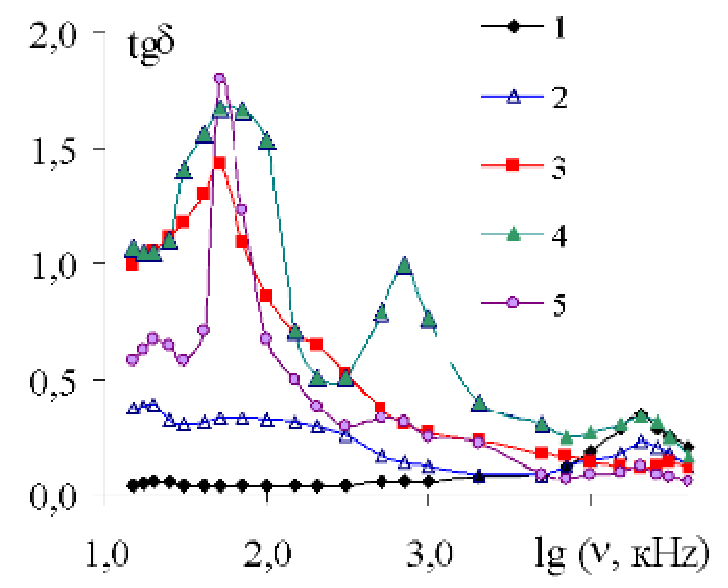

Figure 2. Tangent of dielectrical losses of water of different animals, Semihina

The second law of thermodynamics states that the entropy of any closed system always strives toward an increase, i.e. increase of chaos. Living organisms are open systems, inside them entropy decreases andorder increases. In information theory, entropy is a measure for insufficiency of information in a physical system and is a function of probability. Entropy is infinite if probability is zero. According to Steven Hawking, the second law of thermodynamics defines that the states of chaos significantly outnumber the states of order. He makes the assumption that in the beginning a system has a small number of orderly states. Over time, this system develops according to natural laws and its states change. At later stages, the states of chaos increase in number. Eventually, its states of chaos increase and so does entropy. He uses as an example the computer's memory, which is based on the binary numeral system. The direction of time in which the computer saves the past is the same direction in which disorder increases.

In the 19th century the French scientist Brillouin defined entropy in information systems. On the grounds of biophysical information flows from the living creatures to the environment and vice versa, information properties and the entropy of water in living organisms are changed (Ignatov, Antonov, 1998). Biophysical parameters are introduced for a change of the average energy of the hydrogen bonds in water. "Informationability" of water is related to decrease of entropy in the restructuring of water molecules as a result of external influence (Ignatov, Antonov, 1998). In 1985 the Russian scientist Dulnev measured the parameters of chaos and order as parameters of entropy for one component in a living organism. Yet the living cell is multiparameter with regard to biophysical parameters (Ignatov, 2011).

\subsection{The reasons for aging}

In 1963 Orgel demonstrated that the aging process is associated with the synthesis of proteins. The accumulation of errors in protein synthesis increases exponentially with age (Figure 3). Cells taken from adults show reduced transcription or transmission of information from DNAto RNA. Therefore the likelihood of cancer increases with age.

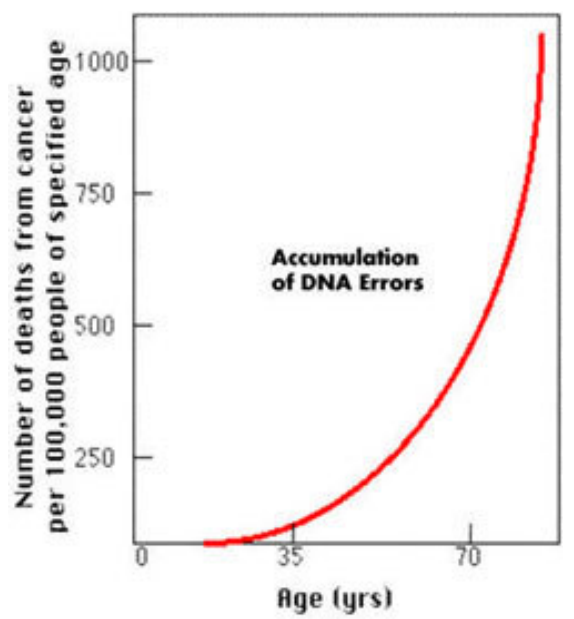

Figure 3. Probable dependence between accumulated DNA errors and cancer mortality 
The best chances of longevity have these people who have direct relatives - long-living people. Experimental data show that the aging process can be slowed down by reducing calories with diet. For example, rats that eat lean food live on average 1.5 times longer than those who eat more fat. (Weindruch R. et al., 1986).

\subsection{Water - the base of life and longevity}

When considering the question of longevity, one has to analyze which are the areas inhabited by the largest number of long-lived people and what are the reasons for that.

In the process of development of each organism, water plays an important part - fundamental matrix of life with a structure, chemical composition and ability to store and transmit information. The human body consists

$50-55 \%$ of water depending on the age and gender. Water is part of the composition of the organism's physiological liquids and plays the role of an internal medium where vital biochemical processes function with the participation of ferments. Because of this, water carries information in the organism regarding aging and longevity.

Human experience shows that long-living people inhabit mainly high mountain areas. In the Russian North there are a lot more centenarians compared to other parts of Russia. (Berdishev, 1960).

Human experience shows that long-living people inhabit mainly high mountain areas. In the Russian North there are a lot more centenarians compared to other parts of Russia. One explanation for this is that the water there contains less deuterium molecules (Berdishev, 1960).

From 1960 to 1965 the scientist Berdishev from the Tomsk Medical Institute studied centenarians in Yakutia and Altai. He linked the longevity of the Yakuts and the Altaians with the use of water from glaciers formed earlier than the Greenland ones. It is well known that most of the Russian centenarians live in Dagestan and Yakutia - 353 and 324 persons per million inhabitants. This figure for Russia is only 8 people. According to Berdishev, defrosted water has a beneficial effect on cellular metabolism. Besides, these people as well as the Buryats, drink mountain water obtained after the melting of ice.

The report's author considers the possibility of extending human life and reducing errors in transcription and replication of DNA in the synthesis of proteins depending on the water we drink.

Cold mountain water has crystal-like structure and consists of water clusters. With the melting of ice only $15 \%$ of hydrogen bonds are destroyed. In this way mountain water contains ice microcrystals. It is quite possible that for this reason defrosted water is beneficial for the body and improves the permeability of cell membranes (23)

\subsection{Spectral analyses with methods NES, DNES and IR}

Studies were performed on a $1 \%$ solution of blood serum with the method of spectral analysis of a nonequilibrium energy spectrum (NES) and differential non-equilibrium energy spectrum (DNES), Antonov@ (1995), Ignatov@ (1998). The samples with blood serum from clynical laboratory were divided into 2 groups of people between 50 and 70 years of age. The first control group consisted of people in excellent health. The second group consisted of people in a critical state and suffering from malignant tumors. The average energy of hydrogen bonds among water molecules in the blood serum was examined as a biophysical parameter. The result was obtained as a difference between the spectrum of $1 \%$ solution of blood serum (NES) and a control sample with deionized water (NES). This spectrum is DNES. The first group obtained the result is $\Delta \mathrm{E}_{\mathrm{H} . . . \mathrm{O}}=-9.1 \pm 1,1$ $\mathrm{meV}$, and the second -

$\Delta \mathrm{E}_{\mathrm{H} . . \mathrm{O}}=-1.6 \pm 1,1 \mathrm{meV}$.

There is statistical difference between the two groups of results according to the t-criterion of Student at level $\mathrm{p}<0,05$.

Water in the human body has a spectrum for the life like information bearer. For the control group of healthy people the value of the spectrum of the largest local maximum is at $-0.1387 \mathrm{eV}$ or at a wavelength of $8.95 \mu \mathrm{m}$

For the group of people in a critical state and the patients with malignant tumors, the values of the spectrum of the largest local maximums shift to lower energies compared with the control group.

A study of blood serum was performed by the method of infrared spectral analysis (Krasnov, Gordetsov, 2009) (24). The following extremums in the spectrum of absorption were received - 8.55, 8.58, 8.70, 8.77, 8.85, $9.10,9.35$ and $9.76 \mu \mathrm{m}$. The resulting extremum at $8.95 \mu \mathrm{m}$ (Ignatov, 2012) is close to the one obtained by Russian scientists at $8.85 \mu \mathrm{m}$. In the control group of healthy people, the function of distribution according to energy $\mathrm{f}(\mathrm{E})$ at $8.95 \mu \mathrm{m}$ has an average value of $75.3 \mathrm{eV}^{-1}$. In the group of people in critical condition this value is $24.1 \mathrm{eV}^{-1}$. The confidence level of the obtained results is $\mathrm{p}<0.05$ by the t-criterion.

In 1992 Antonov performed experiments with the impact on tumor cells of a mouse in water. There was a decrease of the spectrum compared with the control sample of cells from a healthy mouse. Decrease was also observed in the spectrum of the blood serum of terminally ill people to that of healthy people. With the age increase of long-living blood relatives, the function of distribution according to energies at $-0.1387 \mathrm{eV}$ decreases. 
With this group a result was obtained at DNES is $\Delta \mathrm{E}_{\mathrm{H} \ldots \mathrm{O}}=-5.5 \pm 1,1 \mathrm{meV}$ at an age difference of $20-25$ years compared with the control group.

Most of the long-living people in Bulgaria inhabit the Rhodope Mountains. One of the mountain waters, which have a spectrum similar to the spectrum of healthy people blood serum in $8.95 \mu \mathrm{m}$, is the water in the Rhodopes. The mountain waters from Teteven, Boyana, etc., have similar parameters.

\subsection{Comparative analysis between Orgel's chart and the number of long-living people in mountain municipalities in Bulgaria}

Figure 4 shows the interrelation between the year of birth of long lived people (age) and their number (Smolyan municipality, Bulgaria). The dependence between the number of the long-lived peopley and centenarians in Smolyan Municipality and the years of their age is close to exponential. (25).

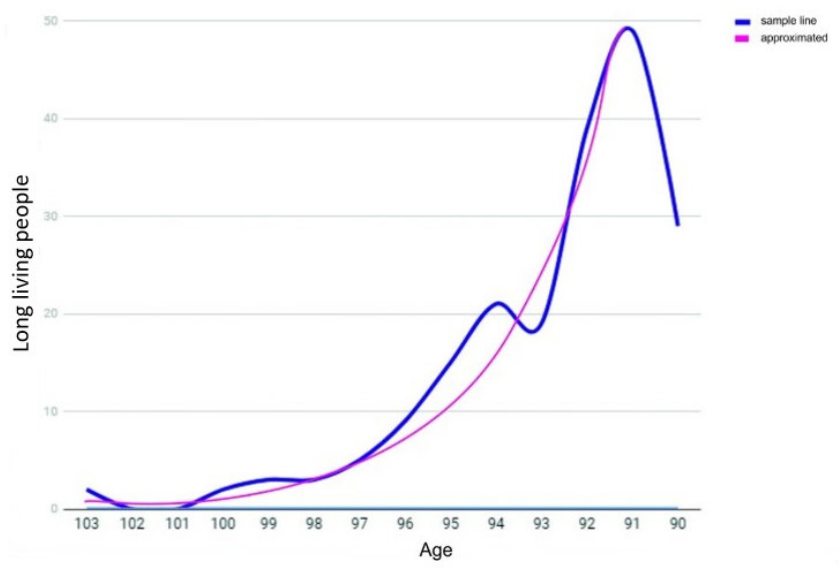

Fugure 4 Interrelation between the year of birth of long-living people (age) and their number in Smolyan municipality, Rhodope Mountains, Bulgaria.

The research shows that more than $90 \%$ of long living people and centenarians in Smolyan Municipality are in excellent health status. Hadzhihristev gives data that more than $70 \%$ have heredity and from the data of the author more than $80 \%$. From the research in 6 municipalities from the regions Lovech, Pleven and Plovdiv, Bulgaria this percent is 40 .

On Figure 5 is given comparative analysis between the number of long-livers and centenarians, and their age in municipality of Smolyan (curve B, violet color) compared with another mountain municipality of Teteven, region of Lovech (curve A, green color). The two municipalities are populated with between 20000 and 40000 people.

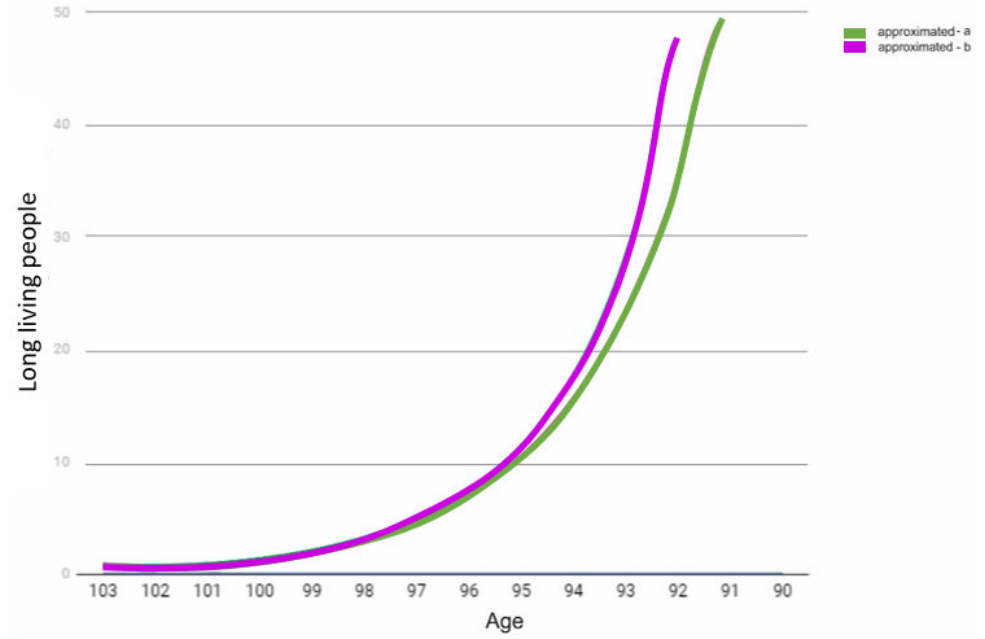

Figure 5. Comparative analysis between the number of long-livers and centenarians, and their age in Municipality of Smolyan, Rhodope Mountains (curve B, violet color) compared with municipality of Teteven, region of Lovech (curve A, green color)

The slope of the chart for long-living people in municipality of Smolyan is lesser than the one for the municipality of Teteven, region of Lovech. That is a proof for a decrease in error rate in DNA replication. It is defined by approximately $40 \%$ higher percentage of heritable long-living people and by the environmental 
factors. In 2014 was created the estimation method by Ignatov and Mosin.

Orgel published the data that the aging process is ssociated with the synthesis of abnormal proteins. Figure

3 shows Orgel's results on the interrelation between age and number of cancer patients.

The accumulation of errors in synthesis of abnormal proteins increases exponentially over time with age. Cells taken from elderly people show the reduced levels of transcription or transmission of information from DNA to RNA. Therefore, the probability of cancer increases with age. The interrelation between the number of Bulgarian long living people and centenarians in the mountainous municipality of Smolyan and their age is close to exponential.

\section{Conclusions and discussion}

The author shows analyses for dependence between entropy and time of life of organisms,

In frame of this project 430 people living in the municipalities of Teteven, Yablanitza. Ugarchin, Lukovit, Lovech district; Dolni Dabnik, Pleven district, Kuklen and Rodopi, Plovdiv district, Smolyan (Bulgaria), where the most number of long lived people and their siblings, were studied. They have the same heredity, but have lived under different conditions. Research shows that the direct relationship of man and nature - clean air, natural food from eco-farms and physical activity explains the difference between the larger number of long lived people and centenarians who live in the mountain regions of Bulgaria. Natural mountain and melt water with chemical composition and structure seems to be one of the most important factors for longevity. In Bulgaria, most long lived people and centenarians live in the Rhodope Mountains.

It worth to note that IR-spectrum of mountain water is most similar to the IR-spectrum of blood serum of healthy group of people with a local extremum at $\lambda=8,95 \mu \mathrm{m}$. Studying of human blood serum by NES and DNES-methods show that by measuring the average energy of hydrogen bonds among $\mathrm{H}_{2} \mathrm{O}$ molecules and the distribution function of $\mathrm{H}_{2} \mathrm{O}$ molecules on energies it is possible to analyse a vital state status of a person and associated life expectancy. There are new proofs for biophysical and biochemical effects of calcium, magnesium, sodium, zinc and manganese on the vital balance, health and longevity (Ignatov, Mosin, 2016).

The optimal composition with $\mathrm{Ca}$ and $\mathrm{Mg}$ ions has the water in New Scotia, Canada. There are living big number of hypercentenarians (more than 110 years old).

\section{References}

Antonov, A. (1995) Research of the Non-equilibrium Processes in the Area in Allocated Systems, Dissertation thesis for degree "Doctor of physical sciences", Blagoevgrad, Sofia.

Berdishev, G, (2002) - Articles Prion as a New form of Life, 2 (3).

Burger, O., Baudish, A\&Vaupel, J. W. (2012) Human Mortality Improvement in Evolutionary Context, $P N A S$, 109 (44): 18210-18214.

Dulnev, G. N., et al. (1975) Minimum Rate of Entropy Production Variational Formulation end Preferred Mode of Motion in Thermal Instability, In Temational Journal of Engineering Science, 13 (9/10), 881-888.

Gluhchev, G., Ignatov, I., Karadzhov, S., Miloshev, G., Ivanov, N.\&Mosin, O.V. (2015) Electrochemically Activated Water. Biophysical and Biological Effects of Anolyte and Catholyte as Types of Water, Journal of Medicine, Physiology and Biophysics, 10, 1-17.

Howard, C. \& Hopps (1986) Chemical Qualities of Water that Contribute to Human Health in a Positive Way, Science of the Total Environment, 54: 207-216.

Howking, S. (1988) A Brief History of Time, Bantam Books.

Ignatov, I., Antonov, A.\&Galabova, T. (1998) Medical Biophysics - Biophysical Fields of Man, First World Congress for Global Health, EWEI, Manila.

Ignatov, I. (2010) Which Water is Optimal for the Origin (Generation) of Life? Euromedica, Hanover, 34-37.

Ignatov, I. (2011) Entropy and Time in Living Organisms, ArchivEuromedica, Hanover, 1st\&2nd Edition, 74-75.

Ignatov, I. (2018) Research of the Factors of Health and Longevity of for the Population in Bulgaria, Bulgarian Journal of Public Health, 10 (1), 34-50..

Ignatov, I., Mosin, O. V.\&Naneva, K. (2012) Water in the Human Body is Information Bearer about Longevity. Euromedica, Hanover: 110-111.

Ignatov I. (2012) Water in the Human Body is Information Bearer about Longevity, Conference on the Physics, Chemistry and Biology of Water, NY, Vermont Photonics.

Ignatov, I., Mosin, O.V.\&Velikov, B. (2014) Longevity Factors and Mountain Water of Bulgaria in Factorial Research of Longevity, Journal of Medicine, Physiology, Biophysics, 1:13-33.

Ignatov, I., Mosin,O.V., Velikov, B., Bauer, E.\&Tyminski, G. (2014) Research of Longevity Factors and Mountain Water as a Factor in Teteven Municipality, Bulgaria, Journal of Medicine, Physiology and Biophysics, 2: 37-52.

Ignatov, I. (2018) Research of the Factors of Health and Longevity of for the Population in Bulgaria, Bulgarian Journal of Public Health, 10 (1), 34-50. 
Ignatov, I.\&Mosin, O.V. (2016) Effects of Calcium, Magnesium, Zinc and Manganese in Water on Biophysical and Biochemical Processes in the Human Body, Journal of Medicine, Physiology and Biophysics, 25, 45-63.

Ignatov, I., Mosin, O. V. (2013) Modeling of Possible Processes for Origin of Life and Living Matter in Hot Mineral and Seawater with Deuterium, Journal of Environment and Earth Science, 3 (14), 103-118.

Ignatov, I. \&Mosin, O. V. (2014) Nature of Haydrogen Bonds in Liquids and Crystals. Ice Crystal Modifications and Their Physical Characteristics, Journal of Medicine, Physiology and Biophysics, 4: 58-80.

Ignatov I.\&Mosin O.V. (2013) Possible Processes for Origin of Life and Living Matter with modeling of Physiological Processes of Bacterium Bacillus Subtilis in Heavy Water as Model System, Journal of Natural Sciences Research, 3(9), 65-76.

Krasnov, V.V. \& Gordetsov, A.S. (2009) Infrared Spectral Analysis of Blood Serum as Level of Disturbances of Metabolic Processes in Infusion Children Pathology, Clinical Medicine: 83-94 [in Russian].

Mosin, O. V.\&Ignatov, I. (2011) Water - Substance of Life, Consciousness and Physical Reality, Natural Sciences, 17 (11), 9-21.

Orgel, L. (1963) The Maintenance of the Accuracy of Protein Synthesis and its Relevance to Aging, Biochemistry, 49: 517-521.

Semihina, LP, (2006) Low-frequency Dielkometriya Vortex Liquids in Weak Electric Fields, Dissertation, Tyumen. 15.

Prigogine, I. (1955) Introduction to Thermodynamics of Irreversible Processes, Charles C. Thomas Publisher, Springfield, Illinois

Weindruch R et al. (1986) The Retardation of Aging in Mice by Dietary Restriction: Longevity, Cancer, Immunity and Lifetime Energy Intake, J Nutr, 116 (4):641-54. 